\title{
Effect of Availability Factor Threshold and Clustering Gap on Performance of Clustering Mechanisms for Multi-cluster Mobile Ad Hoc Networks
}

\author{
Aqeel A. Siddiqui \\ Ericsson Inc. \\ 740 East Campbell Road \\ Richardson, TX 75081
}

\author{
Ravi Prakash \\ Department of Computer Science \\ University of Texas at Dallas, \\ Richardson, TX 75083
}

\begin{abstract}
Mobile ad hoc networks consist of mobile nodes communicating with each other without the presence of any fixed network infrastructure. Existing mechanisms for cluster formation, e.g. node-id based, and degree based, focus on a particular characteristic of the network. The authors have presented earlier a unified mechanism for cluster formation, which not only encompasses existing mechanisms, but also provides means to explore new mechanisms. Two key parameters of this unified mechanism are: availability factor threshold, $\mathbf{a}_{\mathrm{TH}}$, and clustering gap, $\Delta \mathrm{g}$, i.e., the time between two consecutive clustering decisions. In this paper we present the results of the experiments conducted to observe the effect of these two key parameters on the performance of clustering mechanisms. We also present a new performance metric, the unserviced index, which indicates the number of nodes not serviced by any clusterhead at a given time.
\end{abstract}

\section{INTRODUCTION}

In a wireless mobile ad hoc network (MANET) a packet may have to traverse multiple wireless links in order to reach destination [1]-[4]. The advantage of MANETs is rapid deployment and dynamic reconfiguration.

In MANETs, the mobile terminals (or nodes) perform routing in addition to conventional role of acting as hosts. There are several routing techniques in the literature, including flooding, Destination Sequenced Distance Vector (DSDV) [5], Ad-Hoc On Demand Distance Vector (AODV) [6], and Dynamic Source Routing protocol (DSR) [7].

A methodology of organizing nodes as clusters is proposed in [8]-[10]. A node in each cluster assumes the role of clusterhead. All clusterheads (along with some gateway nodes) create a backbone network among themselves, thus keeping each clusterhead up to date on the network topology. The effectiveness of this approach lies in the fact that existing routing protocols can be directly applied to the network replacing the subnets by clusters [10]. A survey of routing protocols is presented in [11].

Most existing clustering protocols try to optimize one performance metric at the expense of others. A unified clustering mechanism is presented in [12], which aims at maximizing all the desirable metrics. Two parameters key to this unified approach are, availability factor threshold, and clustering gap. In this paper we present the effect of these two parameters on performance of unified clustering mechanism.
A new performance metric index for the average unserviced time, is also introduced.

\section{MOTIVATION FOR UNIFIED APPROACH}

A lowest-id linked cluster algorithm (LCA) is described in [8], which is a revised version of [9]. Both clustering mechanisms are often classified as node-id based clustering mechanism and suffer from non-uniform load distribution. A highest connectivity (degree) algorithm (HCA) is presented in [4], which is a modified version of [13]. Simulation results show that the HCA is less stable in a dynamic environment. Other approaches of clustering are described in [14]-[18]. HCA also yields non-uniform clusterhead load distribution, though a lower average number of clusterheads.

To improve the stability of a clustering mechanism, the mobility of the nodes should also be considered [12]. Similarly, the time the nodes have spent as a clusterhead, should be considered while making clusterhead decision in order to improve the clusterhead load distribution.

Therefore, [12] provides a unified method of designing a comprehensive clustering mechanism, which combines all of the above mechanisms and provides flexibility for achieving intermediate performance characteristics. It also defines quantitative measures for a set of performance characteristics.

We have investigated two key parameters of [12], the availability factor threshold, $a_{T H}$, and the clustering gap, $\Delta g$, which denotes the time between two network-wide clustering operations. The effect of these two parameters on the performance characteristics is observed and the results are presented. We describe our results for the change in clustering gap and its effect on the service provided to a node by backbone network. We also highlight new findings and explain the rationale behind them.

\section{UNIFIED CLUSTERING MECHANISM}

\section{A. Unified Clustering Model}

Table I lists the parameters of unified clustering model as described in [12]. The availability factor of each node, $a_{i}(t)$, which is used to decide a clusterhead in a neighborhood, is calculated as,

$$
a_{i}(t)=\alpha \cdot \exp \left(-m_{i}(t)\right)+\beta \cdot c_{i}(t)+\gamma\left(1-q_{i}(t)\right)+\lambda(1-i / N),
$$

where $\alpha, \beta, \gamma$, and $\lambda$, are control parameters controlling the 
nature of clustering mechanism such that the sum of these control parameters is always 1 . Note, $\alpha=1$ is mobility-based algorithm $\beta=1$ is connectivity-based algorithm, $\gamma=1$ is clusterhead-time based algorithm, and $\lambda=1$ is node-id based algorithm The clusterheads are determined periodically $(\Delta g)$ using (2) [12],

$$
\begin{aligned}
& y_{i}(t+\Delta t)= \\
& \left\{1-y_{i}(t)\right\}\left\{1-\underset{j=1, j \neq i}{\left.\boldsymbol{u}\left[\sum_{j}^{N} y_{j}(t) l_{i, j}(t)\right]\right\}} \underset{j=1}{\left\{\prod_{j=1}^{i-1)}\right.} \boldsymbol{u}\left[a_{i}(t)-l_{i, j}(t) a_{j}(t)\left(1-v_{j}(t)\right)\right]\right\} \mathrm{x} \\
& N \\
& \left\{\prod\left(1-\boldsymbol{u}\left[l_{i, j}(t) a_{j}(t)\left(1-v_{j}(t)\right)-a_{i}(t)\right]\right)\right\}+ \\
& \text { (i-1) } \quad j=(i+1) \quad N \\
& \left\{y_{i}(t)\right\}\left\{\prod \boldsymbol{u}\left[a_{i}(t)-l_{i, j}(t) a_{j}(t) y_{j}(t)\right]\right\}\left\{\prod\left(1-\boldsymbol{u}\left[l_{i, j}(t) a_{j}(t) y_{j}(t)-a_{i}(t)\right]\right)\right\} \\
& j=1 \quad j=(i+1) \\
& +\left\{1-y_{i}(t)\right\}\left\{\boldsymbol{u}\left[\sum^{N} y_{j}(t) l_{i, j}(t)\right]\right\} \mathrm{x} \\
& \text { (i-1) } \quad j=1, j \neq i \\
& \left\{\prod \boldsymbol{u}\left[a_{i}(t)-l_{i, j}(t)\left(a_{j}(t)+a_{T H}\right) y_{j}(t)\right]\right\} \mathrm{x} \\
& j=1 \\
& \left\{\prod^{N}\left(1-\boldsymbol{u}\left[l_{i, j}(t)\left(a_{j}(t)+a_{T H}\right) y_{j}(t)-a_{i}(t)\right]\right)\right\}
\end{aligned}
$$

where $u(x)=0$, for $x \leq 0$, otherwise 1 .

Three performance characteristics have also been defined in [12] as follows:

- $G(t)$ : Granularity of clusterheads, i.e., the time averaged ratio of number of clusterheads to the total number of nodes of the system, defined as:

$$
G(t)=\frac{1}{N} \sum_{i=1}^{N} q_{j}(t) ; 0 \leq G(t) \leq 1 .
$$

After the ad hoc network operates for some time, the spatial distribution of clusterheads converges to one value, which we denote as, $G$. A lower $G$ is generally desired.

- $S(t)$ : Stability of the system, i.e., the duration for which a clusterhead remains in that state, defined as

$$
S(t)=\frac{1}{N} \sum_{j=1}^{N} s_{j}(t) ; \quad 0 \leq S(t) \leq 1 .
$$

When the stability converges to a value, it is denoted as $S$. A higher $S$ is generally desired.

- $D(t)$ : clusterhead load distribution of the system i.e., the amount of work performed by each clusterhead, defined as

$$
D(t)=1-\frac{1}{N} \sqrt{\sum_{j=1}^{N}\left(q_{j}(t)-G(t)\right)^{2}} \quad ; \quad 0 \leq D(t) \leq 1 .
$$

The converged value of the clusterhead load distribution is

\begin{tabular}{|c|c|}
\hline Parameter & Description \\
\hline $\bar{N} N$ & Total number of nodes. \\
\hline$\Delta g$ & $\begin{array}{l}\text { The interval of time between two clustering } \\
\text { decisions. }\end{array}$ \\
\hline$\Delta t$ & $\begin{array}{l}\text { Minimum interval of time between two } \\
\text { consecutive link updates, } \Delta t \leq \Delta g .\end{array}$ \\
\hline$y_{i}(t)$ & $\begin{array}{l}\text { If node } i \text { is a clusterhead at time } t \text {, then } y_{i}(t)=1 \text {, } \\
\text { otherwise } 0 .\end{array}$ \\
\hline$v_{i}(t)$ & $\begin{array}{l}\text { If node } i \text { has a clusterhead neighbor at time } t \\
\text { then } v_{i}(t)=1 \text {, otherwise } 0 .\end{array}$ \\
\hline$l_{i, j}(t)$ & $\begin{array}{l}\text { If node } i \text { is a neighbor of node } j \text { at time } t \text {, then } \\
l_{i, j}(t)=1 \text {, otherwise } 0 .\end{array}$ \\
\hline$c_{i}(t)$ & Connectivity or degree of node $i$. \\
\hline$m_{i}(t)$ & Mobility of node $i$. \\
\hline$q_{i}(t)$ & $\begin{array}{l}\text { The fraction of time node } i \text { remains a clusterhead } \\
\text { in the last period of time } T \text {. }\end{array}$ \\
\hline$z_{i}(t)$ & $\begin{array}{l}\text { The instability of node } i \text { as a clusterhead, at time } \\
t . \text { It is the number of times the node changes the } \\
\text { role of a clusterhead per hour, measured in the } \\
\text { last period of time } T \text {. }\end{array}$ \\
\hline$s_{i}(t)$ & The stability of node $i, \exp \left(-z_{i}(t)\right)$. \\
\hline$a_{i}(t)$ & The availability factor of node $i$ at time $t$. \\
\hline$a_{T H}$ & Availability factor threshold. \\
\hline
\end{tabular}
denoted as, $D$. A higher $D$ is generally desired.

\section{B. Availability Factor Threshold}

Note in (2) that for a node $i$ to become clusterhead in the presence of a neighboring clusterhead node, the availability factor of the node $i$ should be greater than that of all neighboring clusterhead nodes by $a_{T H}$. This is the availability factor threshold. The availability factor threshold introduces a hysteresis, thus favoring a node to remain clusterhead once it becomes clusterhead. Therefore, the larger the availability factor threshold the higher will be the stability of the system. It may also result in non-uniform clusterhead load distribution. Therefore, the availability factor threshold should be chosen carefully.

TABLE I: PARAMETERS OF UNIFIED CLUSTERING MECHANISM

\section{Clustering Gap}

We assume that the clocks of all nodes are synchronized and nodes can communicate in a synchronous fashion without any interference and collisions, where the execution/communication advances in rounds of duration $\Delta t$. Providing such a mechanism is the responsibility of the link layer and physical layer, details of which are beyond the scope of this paper. The Time Division Multiple Access (TDMA) can be used, in which case, we assume that each round has a duration $\Delta t$ equal to TDMA frame time, and there are as many time slots in a frame as the number of nodes in the system [12].

The clusterhead decisions are made periodically with a time interval of $\Delta g$. This time interval is called as clustering 
gap. The clustering gap is a multiple of $\Delta t$ as shown in Figure 1. The time period $\Delta t$ is fixed.

Neighboring nodes exchange information at each increment $\Delta t$, and may also update the link state, but the clusterhead decisions are delayed until $\Delta g$ after the previous clustering decision. Larger $\Delta g$ is expected to result in higher stability as the clusterhead decisions will be made less frequently. However, this will result in larger number of nodes unserviced by any clusterhead, during the clustering gap. A node is said to be unserviced if it is neither a clusterhead, nor adjacent to a clusterhead.

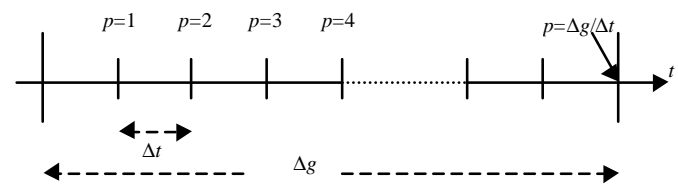

Figure 1: Relationship between clustering gap and $\Delta t$

We define a set of system level parameters, called unserviced index, $U^{p}(t)$. This unserviced index is the average number of unserviced nodes $p \Delta t$ time after each clustering decision in the last $T$ time period. Here $p$ is the position shown in Figure 1. The unserviced index can be mathematically expressed as follows:

$$
U^{p}(t)=\frac{1}{n_{T}} \sum_{x=1}^{n_{T}} \sum_{i=1}^{N}\left(1-v_{i}(t-x \Delta g+p \Delta t)\right) ; 1 \leq p \leq \Delta g / \Delta t .
$$

Where $n_{T}=T / \Delta g$, and $t$ is the time at which clusterhead decisions are made. After the ad hoc network operates for some time, the unserviced index, for each position $p$, converges to one value, which we denote as, $U^{p}$. A lower $U^{p}$ is generally desirable.

\section{EXPERIMENTS AND RESULTS}

To observe the effect of availability factor threshold and clustering gap, we have simulated a mobile ad hoc network of 20 nodes, with service area $1000 \mathrm{~m} \times 1000 \mathrm{~m}$, maximum coverage radius $250 \mathrm{~m}$, and the mobility range $1.5 \mathrm{~m} / \mathrm{s}-4.5 \mathrm{~m} / \mathrm{s}$. The time period $T$, is 8 hours, and the incremental time period $\Delta t$, is $0.1 \mathrm{sec}$.

Two sets of measurements have been performed:

1. Availability factor threshold, $a_{T H}=0,0.1,0.2$, and 0.3 , with clustering gap $\Delta g=0.1 \mathrm{~s}$.

2. Clustering gap $\Delta g=0.1 \mathrm{~s}, 1 \mathrm{~s}$, and $10 \mathrm{~s}$, with availability factor threshold, $a_{T H}=0.2$.

Each node randomly assumes a speed within the given mobility range at the beginning of simulation, and then moves with that constant speed at each time increment in a random direction.

The ad hoc network's operation for a 16 hour duration is simulated (this duration is found to be sufficient, using repeated trials, to get converged values of performance characteristics). Note that the performance is averaged over the last $T$ time period. The performance is determined by simulation, assuming each node has complete information about the network (best case).

The following are the steps of simulation: (i) data initialization, (ii) update availability factor for all nodes, (iii) if time for clustering then determine $y_{i}(t)$ for all nodes using (2), (iv) increment time by $\Delta t$, (v) move all nodes, (vi) repeat steps (ii)-(v) until the end of simulation period, and (vii) determine performance using (3) - (6).

\section{A. Availability Factor Threshold}

Figures 2 and 3 show that the stability increases with the increase of $a_{T H}$. Note in Figure 2, when the availability factor threshold is increased from 0 to 0.1 , the stability $(S)$ increases without any decrease in the clusterhead load distribution $(D)$. Therefore, we suggest that $a_{T H}=0.1$ should be the minimum availability factor threshold, or $a_{T H m i n}$. On the higher side, when the $a_{T H}$ is increased beyond $a_{T H}=0.2$, the performance characteristics remain unchanged. Therefore, $a_{T H}=0.2$ should be the maximum availability factor threshold, or $a_{T H \text { max }}$.

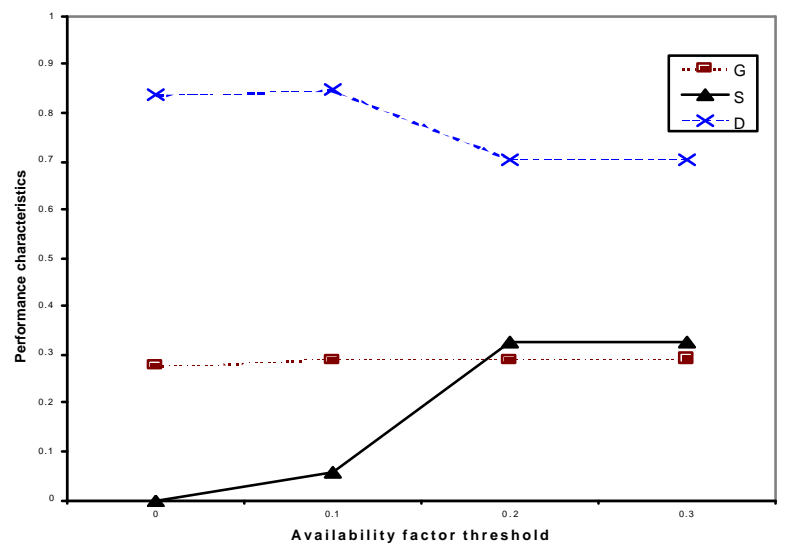

Figure 2: Performance characteristics for connectivity-based algorithm

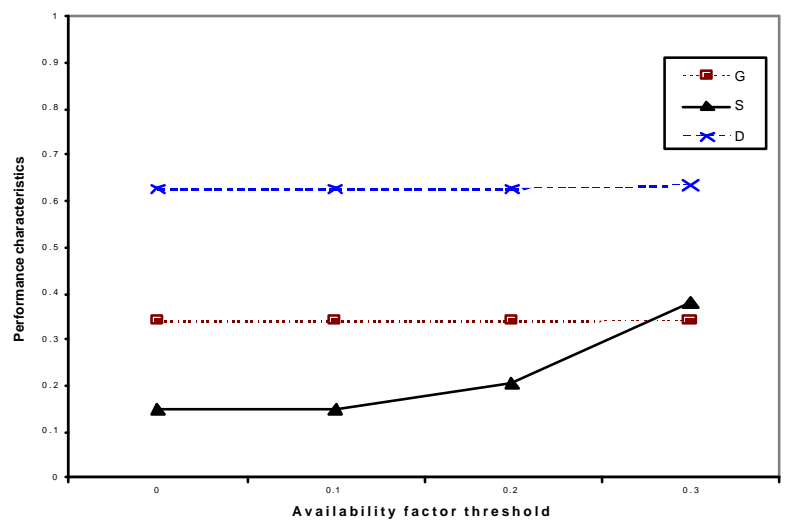

Figure 3: Performance characteristics for node-id based algorithm 
The values of $a_{\text {THmin }}$ and $a_{\text {THmax }}$ are different for each clustering mechanism as could be seen from Figures 2-3 (other mechanisms not shown here due to lack of space). The availability factor threshold for a clustering mechanism should be chosen between these two extreme values. This selection is based on the best acceptable combination of performance characteristics. In some cases these minimum and maximum values of availability factor threshold may not be very obvious. The selection of availability factor threshold is subjective in such cases.

\section{B. Clustering Gap}

Figures 4-7 show the unserviced index $\left(U^{p}\right)$ versus time elapsed since last clustering (which is proportional to the position $p$ introduced in Figure 1). The unserviced index is shown for each type of clustering mechanism and with various values of clustering gap. Note that the larger the time elapsed since last clustering, the larger is the average number of unserviced nodes.

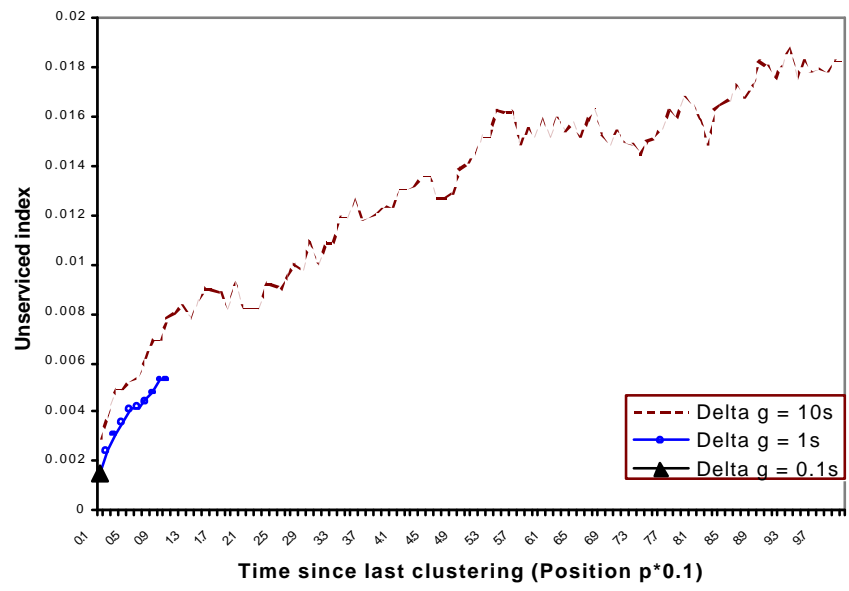

Figure 4: Unserviced index for mobility-based algorithm

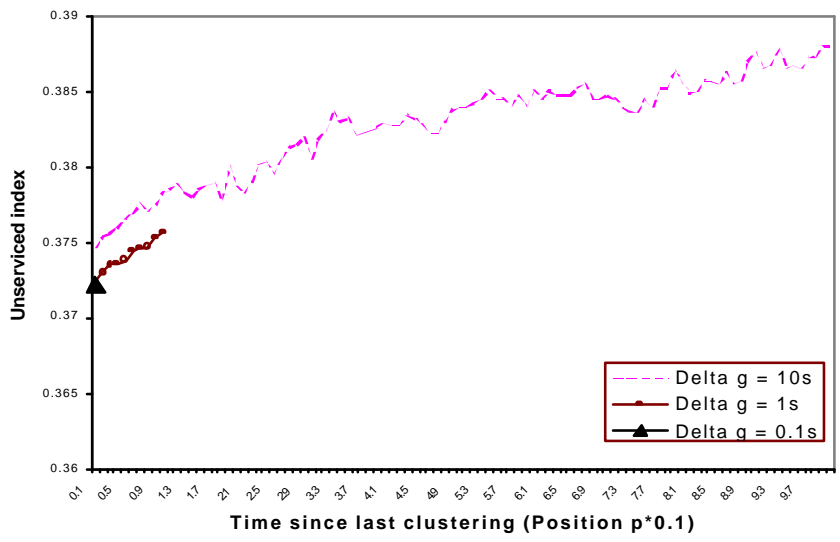

Figure 5: Unserviced index for connectivity-based algorithm

Another interesting observation is that at one time increment $(\Delta t)$ after last clustering (i.e., at $p=1$ ), the higher clustering gap $(\Delta g)$ results in larger number of unserviced nodes. The reason is that the likelihood of a node to change its clusterhead state (i.e., a clusterhead becoming a nonclusterhead and vice versa) at time $t+\Delta g$ is higher for larger $\Delta g$. In (2) the clusterhead decision at time $t+\Delta g$ is made based on the state of nodes at time $t+\Delta g-\Delta t$. As the nodes may change their clusterhead state simultaneously at $t+\Delta g$, the clusterhead decisions are sub-optimal. The larger the clustering gap the less optimal are the clusterhead decisions, resulting in larger number of unserviced nodes at one time increment $(\Delta t)$ after clusterhead decisions are made.

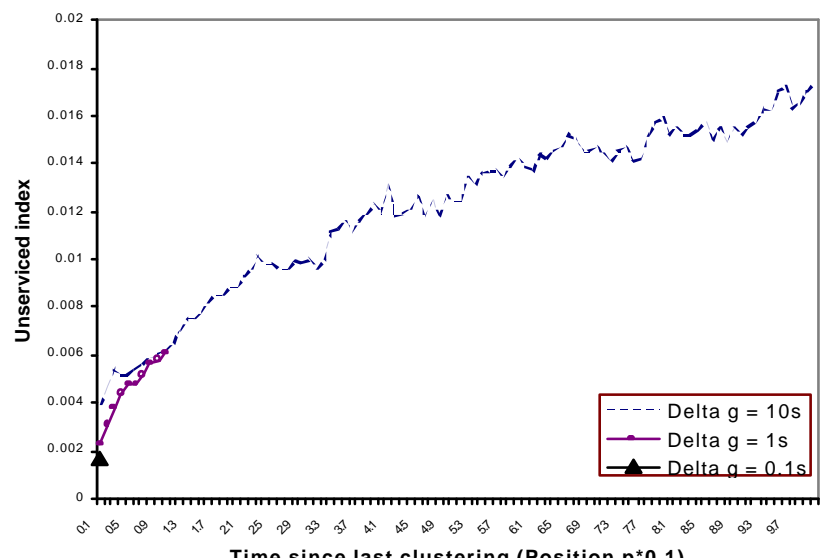

Figure 6: Unserviced indexfor clusterhead time-based algorithm

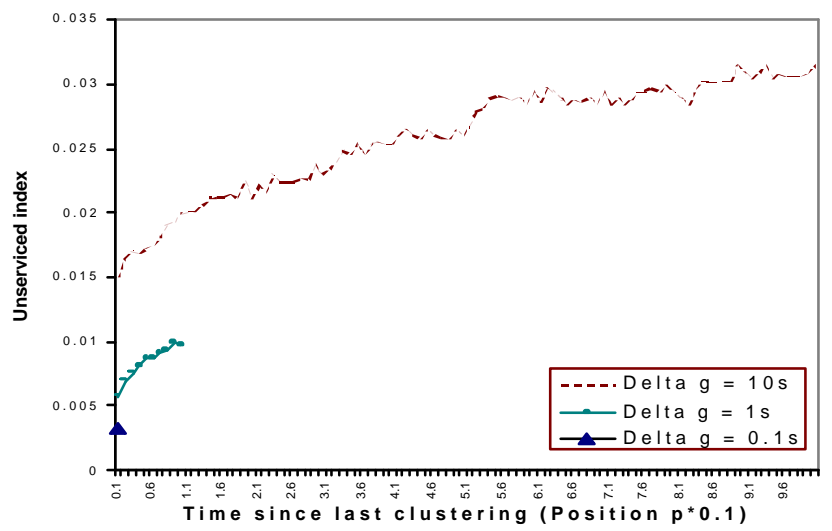

Figure 7: Unserviced indexfor node-id based algorithm

Also, Figures 4-7 illustrate that the connectivity-based algorithm has a significantly higher unserviced index than the other methods. Hence, the connectivity-based method appears to be less suitable than the other methods.

The performance characteristics for different values of clustering gap have also been observed and shown in Tables II-III. It shows that the increase of clustering gap results in increase of stability without significant change in clusterhead load distribution. Therefore, we conclude that the deciding factor for the value of clustering gap is the acceptable value of unserviced index. Of course, if the clustering gap is made 
too large, i.e., comparable to the time period $T$, then it will impact the clusterhead load distribution also.

TABLE II: STABILITY, $S$

\begin{tabular}{||c||c|c|c||}
\hline \multirow{2}{*}{$\begin{array}{c}\text { Clustering } \\
\text { algorithm } \\
\text { based on }\end{array}$} & \multicolumn{3}{|c|}{ Clustering gap $(\Delta g)$} \\
\cline { 2 - 4 } & 0.1 & 1 & 10 \\
\hline \hline Mobility & 0.472 & 0.477 & 0.591 \\
\hline Connectivity & 0.359 & 0.464 & 0.557 \\
\hline CH time & 0.187 & 0.251 & 0.316 \\
\hline Node-id & 0.205 & 0.281 & 0.439 \\
\hline
\end{tabular}

TABLE III: CLUSTERHEADLOAD DISTRIBUTION, $D$

\begin{tabular}{||c||c|c|c||}
\hline \multirow{2}{*}{$\begin{array}{c}\text { Clustering } \\
\text { algorithm } \\
\text { based on }\end{array}$} & \multicolumn{3}{|c|}{ Clustering gap $(\Delta g)$} \\
\cline { 2 - 4 } & 0.1 & 1 & 10 \\
\hline \hline Mobility & 0.645 & 0.654 & 0.651 \\
\hline Connectivity & 0.704 & 0.704 & 0.674 \\
\hline CH time & 0.907 & 0.910 & 0.899 \\
\hline Node-id & 0.626 & 0.626 & 0.626 \\
\hline
\end{tabular}

\section{CONCLUSION}

The effect of availability factor threshold on the performance characteristics is observed. The minimum availability factor threshold provides better stability without sacrificing clusterhead load distribution. The maximum availability factor threshold provides highest stability but with a relatively lower clusterhead load distribution. The availability factor should chosen between these two values.

The performance characteristics tend to improve with the increase in clustering gap, but at a cost of increased unserviced index. Therefore, the acceptable value of unserviced index is the deciding factor for choosing clustering gap.

\section{REFERENCES}

[1] Ram Ramanathan, and Regina Rosales-Hain. Topology Control of Multihop Wireless Networks using Transmit Power Adjustment. IEEE Infocom 2000, pages 404-413.

[2] Ching-Chuan Chiang, and Mario Gerla. Routing and Multicast in Multihop, Mobile Wireless Networks. Proceedings of IEEE Singapore International Conference on Networks (SICON'97), http://www.cs. ucla.edu/NRL/ wireless/PAPER/ Chiang_sicon97.ps.gz

[3] J. Ioannidis, D. Duchamp, and G. Q. M. Jr. IP-based protocols for mobile interworking. In SIGCOM, pages 235-243. ACM, 1991.

[4] Mario Gerla and Jack Tzu-Chieh Tsai. Multicluster, Mobile, Multimedia Radio Network. ACM Journal on Wireless Networks, Vol. 1, No. 3:255-265, 1995.

[5] Charles E. Perkins and Pravin Bhagwat. Highly dynamic Destination-Sequenced Distance-Vector (DSDV) routing for mobile computers. In Proceedings of the SIGCOM '94 Conference on Communications Architecture, protocols and Applications, pages 234-244, August
1994.

[6] Charles E. Perkins, Elizabeth M. Royer, and Samir R. Das. Ad Hoc On Demand Distance Vector (AODV) Routing. Internet draft, draft-ietf-manet-aodv-08.txt, March 2001.

[7] J. Broch, D. B. Johnsson, D. A. Maltz. The Dynamic Source Routing for Mobile Ad Hoc Networks. Internet draft, draft-ietf-manet-dsr-05.txt, March 2001.

[8] Anthony Ephremides, Jeffrey E. Wieselthier, and Dennis J. Baker. A design concept for reliable mobile radio networks with frequency hopping signaling. In Proceedings of IEEE, 75(1) (1987), pages 56-73.

[9] Dennis J. Baker and Anthony Ephremides. The Architectural Organization of a Mobile Radio Network via a Distributed Algorithm. IEEE Transactions on Communications, Vol. Com-29, No. 11, November 1981, pages 1694-1701.

[10] P. Krishna, N. H. Vaidya, M. Chatterjee, and D. K. Pradhan. A Cluster-based Approach for Routing in Dynamic Networks. In SIGCOMM Computer Communications Review (CCR), 1997. http://www.acm. org/sigs/sigcomm/ccr/archive/1997/apr97/ccr-9704krishna.pdf

[11] S. Ramanathan, and M. Steenstrup. A Survey for Routing Techniques for Mobile Communication Networks. In Mobile Networks and Applications 1, 1996, pages 89-104

[12] A. Siddiqui, and R. Prakash. Modeling, Performance Measurement, and Control of Clustering Mechanisms for Multi-cluster Mobile Ad Hoc Networks. Technical Report UTDCS-16-01. http://www. utdallas.edu/ ravip/ paper.list. html

[13] Abhay K. Parekh. Selecting routers in ad-hoc wireless networks. In ITS 1994.

[14] Prasun Sinha, Raghupathy Sivakumar, and Vaduvur Bharghavan. CEDAR: a Core-Extraction Distributed Ad hoc Routing Algorithm. Infocom 99. http://www. ieee-infocom.org/1999/papers/02a_04.pdf

[15] R. Krishnan, R. Ramanathan, and M. Steenstrup. Optimization Algorithms for Large Self-Structuring Networks. Infocom 99. Eighteenth Annual Joint Conference of the IEEE Computer and Communications Societies. Proceedings. IEEE, Volume: 1, 1999, pages $71-78$ vol.1.

[16] Barry M. Leiner, Donald L. Nielson, and Fouad A. Tobagi. Issues in Packet Radio Network Design. Proceedings of the IEEE, Vol. 75, No. 1, January 1987, pages 6-20.

[17] Nachum Shacham, and Jil Westcott. Future Directions in Packet Radio. Proceedings of the IEEE, Vol. 75, No. 1, January 1987, pages 83-99.

[18] A.D. Amis, R. Prakash, T. H. P. Vuong, and D. T. Huynh. Max-Min D-Cluster Formation in Wireless Ad Hoc Networks. In Proceedings of Infocom 2000. http://www.ieee-infocom.org/2000/papers/281.ps 
